\title{
Relationship of Intraoperative Anatomical Landmarks, the Scapular Plane and the Perpendicular Plane with Glenoid for Central Guide Insertion during Shoulder Arthroplasty
}

\author{
Jung-Han Kim, Young-Kyoung Min \\ Department of Orthopedic Surgery, Inje University Busan Paik Hospital, Inje University College of Medicine, Busan, Korea
}

Background: This study was undertaken to evaluate the positional relationship between planes of the glenoid component (the scapular plane and the perpendicular plane to the glenoid) and its surrounding structures.

Methods: Computed tomography (CT) images of both shoulders of 100 patients were evaluated using the 3-dimensional CT reconstruction program (Aquarius ${ }^{\circledR}$; TeraRecon). We determined the most lateral scapular bony structure of the scapular plane and measured the shortest distance between the anterolateral corner of the acromion and the scapular plane. The distance between the scapular plane and the midpoint of the line connecting the posterolateral corner of acromion and the anterior tip of the coracoid process (fulcrum axis) was also evaluated. The perpendicular plane was then adjusted to the glenoid and the same values were re-assessed.

Results: The acromion was the most lateral scapular structure of scapular plane and perpendicular plane to the glenoid. The average distance from the anterolateral corner of the acromion to the scapular plane was $10.44 \pm 5.11 \mathrm{~mm}$, and to the plane perpendicular to the glenoid was $9.55 \pm 5.13 \mathrm{~mm}$. The midpoint of fulcrum axis was positioned towards the acromion and was measured at $3.90 \pm 3.21$ $\mathrm{mm}$ from the scapular plane and at $3.84 \pm 3.17 \mathrm{~mm}$ from the perpendicular plane to the glenoid.

Conclusions: Our data indicates that the relationship between the perpendicular plane to the glenoid plane and its surrounding structures is reliable and can be used as guidelines during glenoid component insertion (level of evidence: Level IV, case series, treatment study).

(Clin Shoulder Elbow 2018;21(3):113-119)

Key Words: Glenoid; Scapular plane; Glenoid plane; Total shoulder arthroplasty; Fulcrum axis

\section{Introduction}

Total shoulder arthroplasty is an effective surgical treatment for end-stage osteoarthritis of the shoulder joint. ${ }^{1-6)}$ The frequency of shoulder arthroplasty has been on the rise, and clinical outcomes have improved ${ }^{1,7,8)}$ with advances in implant techniques and understanding of shoulder biomechanics. Glenoid component insertion is an important technique in total shoulder arthroplasty. ${ }^{8)}$ However, preparing the side of the glenoid and glenoid component insertion remains challenging. ${ }^{9)}$ Malalignment of the glenoid component leads to eccentric loading, shift of vector force, increased contact pressure and rim-loading, increased stress at the implant-bone or bone-cement interface and the glenoid bone stock, resulting in an increased rate of glenoid failure in the long term. ${ }^{10,11)}$ The most common cause of revisions of total shoulder arthroplasties are reportedly due to glenoid wear, osteolysis, and loosening. ${ }^{12-14)}$ The accurate placement of the glenoid component is therefore an important technical goal in shoulder arthroplasty. ${ }^{15)}$ When sufficient exposure of the required glenoid is attained, the appropriate position and angle for insertion is possible. However, the field of operation for glenoid surgery is very narrow; moreover, the reamer head

Received May 13, 2018. Revised June 12, 2018. Accepted June 20, 2018.

Correspondence to: Young-Kyoung Min

Department of Orthopedic Surgery, Inje University Busan Paik Hospital, Inje University College of Medicine, 75 Bokji-ro, Busanjin-gu, Busan 47392, Korea

Tel: +82-51-890-6129, Fax: +82-51-892-6619, E-mail: dazuri@hanmail.net, ORCID: https://orcid.org/0000-0002-8076-7265

IRB approval: Inje University Busan Paik Hospital (No. 15-0005).

Financial support: None. Conflict of interests: None. 
is large and hinders the field, making it difficult to attain the correct position and angle when reaming. ${ }^{3)}$ In addition, it is more difficult in revision cases and in patients with osteoarthritis, soft tissue contractures or posterior glenoid wear. ${ }^{7,16-18)}$ In most situations, the surgeon has to rely on subjective feel and personal experience to match the intraoperative findings with the preoperative images, without any additional reference to the correct angle of the scapula position. Hence, the surgeon needs to rely on experience to evaluate the inclination of the glenoid and the correction angle in a restricted field. ${ }^{3,16,19-21)}$ Moreover, posterior glenoid wear is often present in the osteoarthritic shoulder, ${ }^{22-24)}$ resulting in intact articular cartilage and subchondral bone anteriorly, leading to increased retroversion of the diseased glenoid. Depending on the degree angle of posterior wear, numerous studies have suggested different treatment options for glenoid resurfacing. ${ }^{10,11,17,25)}$ However, in the surgical field there is no qualitative guideline for evaluating the amount of eccentric reaming. ${ }^{26)}$ Failure of the surgeon to recognize posterior wear during shoulder arthroplasty may lead to implantation of the glenoid component in excessive retroversion, or reaming in a manner that will not correct the posterior wear. ${ }^{11,25)}$ Although few reports have suggested techniques for correct glenoid centering, these techniques were not reproducible. Moreover, these reports did not have objective references and evaluations. Preoperative computed tomography (CT) evaluates the deformities and predicts the glenoid center, but the intraoperative application of CT results is difficult and inaccurate. Navigation systems are not available in all facilities and countries. Moreover, the procedure is not familiar to many surgeons, and has the added disad- vantage of increase in the operation time and cost due to the instruments used. ${ }^{21,27)}$ Therefore, there is a high requirement for an easily and accurately identifiable intraoperative anatomical landmark for proper glenoid centering. This study was therefore undertaken to evaluate the positional relationship between the plane of the glenoid component and its surrounding structures. We believe this data may be useful as anatomical guidelines for intraoperative glenoid component insertion.

\section{Methods}

After obtaining approval from the Institutional Review Board of Inje University Busan Paik Hospital (No. 15-0005), 100 patients who had CT of both shoulders between January 2010 to December 2014 were selected for the study. Patients with previous trauma and operative history of the shoulder, osteoarthritis of the shoulder, or age over 50 years were excluded. Totally, 61 men and 39 women were enrolled, having mean age $39.7 \pm 3.1$ years (range: 20-49 years). Using the radiographic evaluation program of Aquarius $^{\circledR}$ (TeraRecon, San Mateo, CA, USA), we set up a $2 \times 2$ layout workstation screen and used the mouse interface, including the functionality of scroll, pan, zoom, windowing, angle, and distance. The plane passing through the angular inferior, trigonum scapulae, and glenoid center of the scapula was defined as the scapular plane. The axial and coronal views were adjusted to display and mark the trigonium scapulae and glenoid centers, and the coronal and sagittal views were adjusted to display and mark the trigonum scapulae. The scapular plane passing through these 3 marked points was then obtained (Fig.
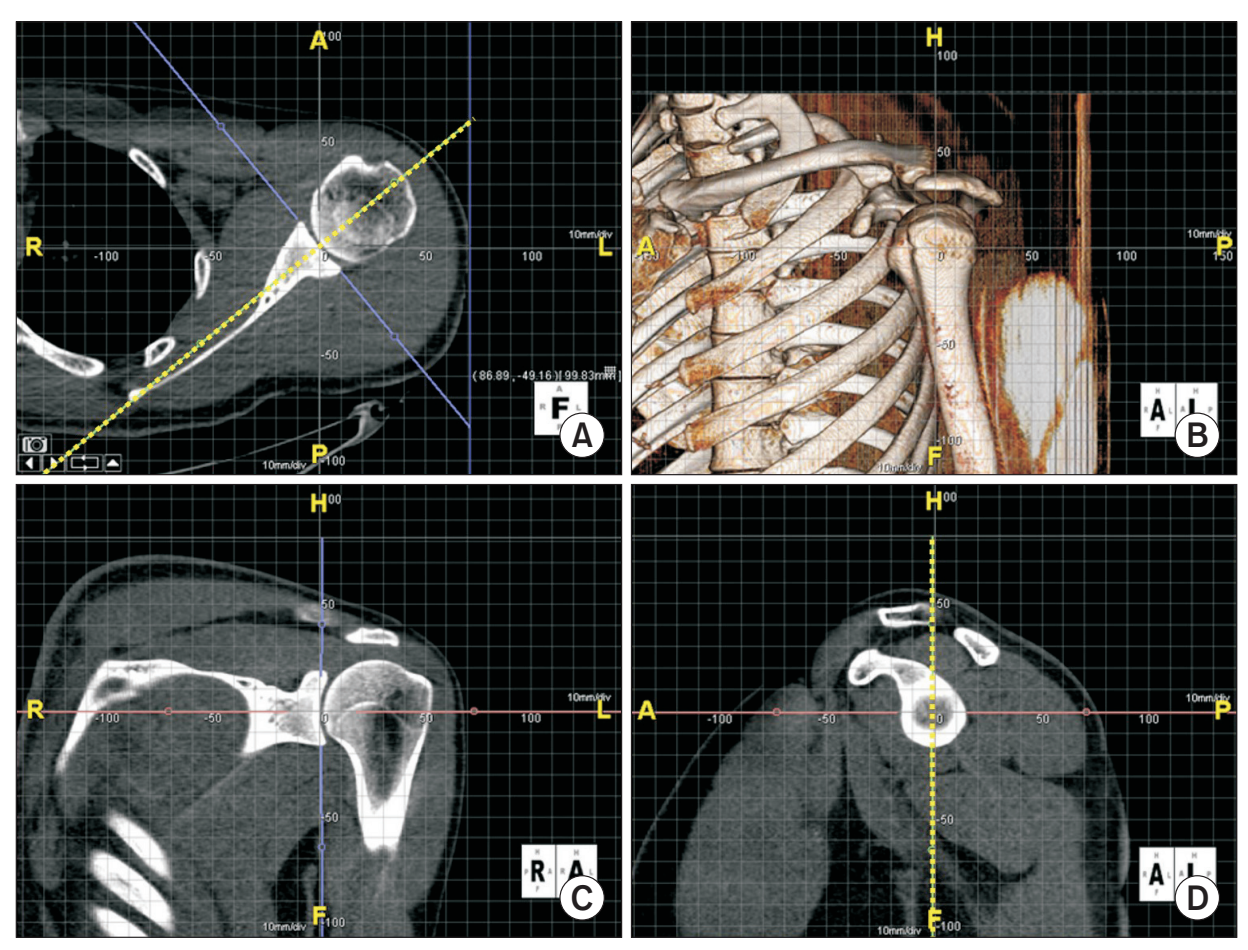

Fig. 1. Reconstruction to scapular plane (dash lines). (A) Axial image: the plane passing through the scapular medial border and the glenoid center is obtained. (B) Threedimensional reconstructed volume rendering model. (C) Coronal image: the plane passing through the trigonum scapulae and the glenoid center is obtained. (D) Sagittal image: ensures that the scapular plane passes the glenoid center. 
1). The most lateral scapular bony structure of the scapular plane was identified. This structure included the acromion, acromioclavicular (AC) joint and the lateral end of clavicle (Fig. 2). Next, we adjusted the axial image through the anterolateral corner of the acromion and measured the shortest distance between the anterolateral corner of the acromion and the scapular plane. Because the posterolateral corner of the acromion and the anterior tip of the coracoid can be palpated by hand during surgery, we defined the fulcrum axis as a line passing through two anatomical points. The relationship between the line passing through these two points and the scapular plane was investigated; using the Aquarius ${ }^{\circledR}$, we sent the 3-dimensional (3D) volume rendering (VR) image to the screen. After drawing a line connecting the posterolateral corner of the acromion with the anterior tip of the coracoids, we marked the midpoint of the fulcrum axis line and a line passing through the point of fulcrum axis on the scapular plane. The distance between the two points was then measured in millimeters (coracoid side [-], acromion side [+]) (Fig. 3). We also adjusted each plane of the axial, coronal, and sagittal views perpendicular to the glenoid at the glenoid center level and identified the most lateral scapular bony structure of the perpendicular plane to glenoid (Fig. 4). A second axial cut retaining the anterolateral corner of the acromion was selected and we measured the shortest distance between the point and the perpendicular plane to the glenoid plane. Similar to the previous protocol, the 3D VR images were sent to the screen using the Aquarius $^{\circledR}$. A line was then drawn to connect the posterolateral corner of the acromion to the anterior tip of the coracoids, and the distance was measured in millimeters from midpoint of the line to the perpendicular plane to glenoid plane (coracoid side [-], acromion side [+]). All measurements were individually carried out by 2 orthopedic surgeons; all measurements were taken twice and the average values were calculated. Statistical analysis was achieved using the IBM SPSS ver. 22.0 statistics software (IBM Co., Armonk, NY, USA). The inter-observer reliability and intraobserver reliability were evaluated by a reliability test. The $\mathrm{p}$ values less than 0.05 were defined as statistically significant.

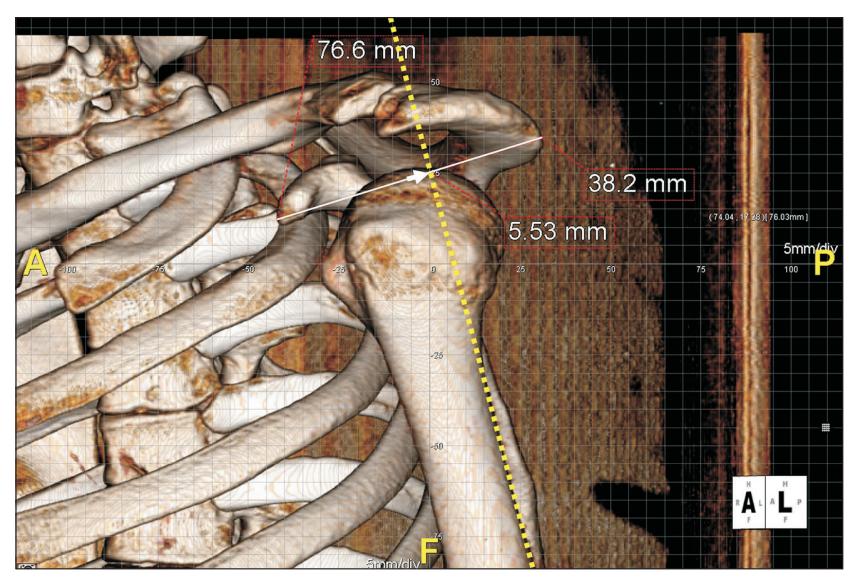

Fig. 3. By using the programs, 3-dimensional reconstructed images were transferred to the screen. After drawing the fulcrum axis (white line; defined as a line connecting the posterolateral corner of the acromion and the anterior tip of the coracoids) on the screen, the middle point was marked. Distance between the middle point and the scapular plane (dash line) was measured in millimeters (coracoid side [-], acromion side [+]). In this figure, the scapular plane passes at $5.5 \mathrm{~mm}$ toward the acromion.
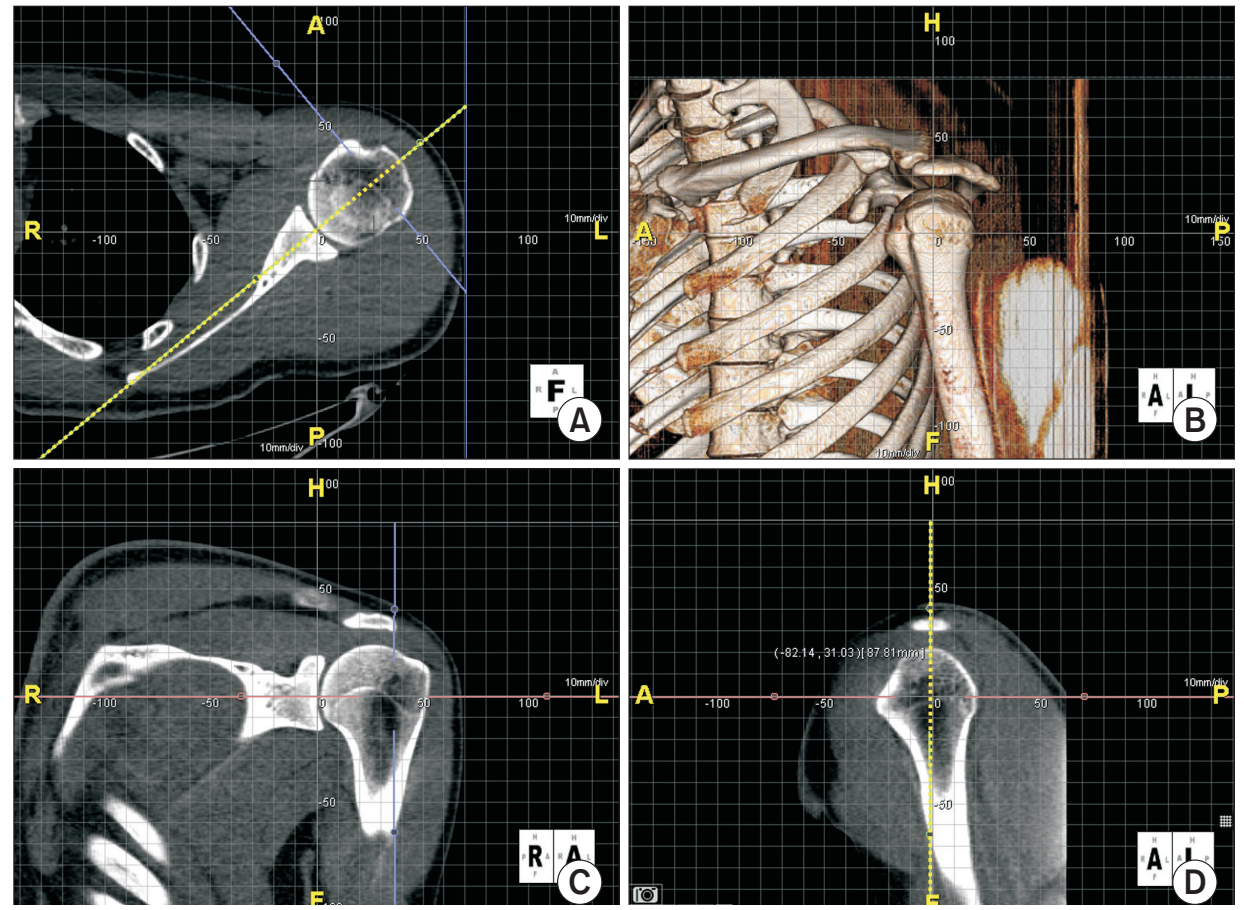

Fig. 2. The most lateral scapular bony structure of the scapular plane (dash line). (A) Axial image: the scapular plane is fixed. (B) Three-dimensional reconstructed volume rendering model. (C) Coronal image: sagittal image is moved to the lateral side while viewing the coronal image. (D) Sagittal image: lateral most side of the scapular plane passes through the acromion. 

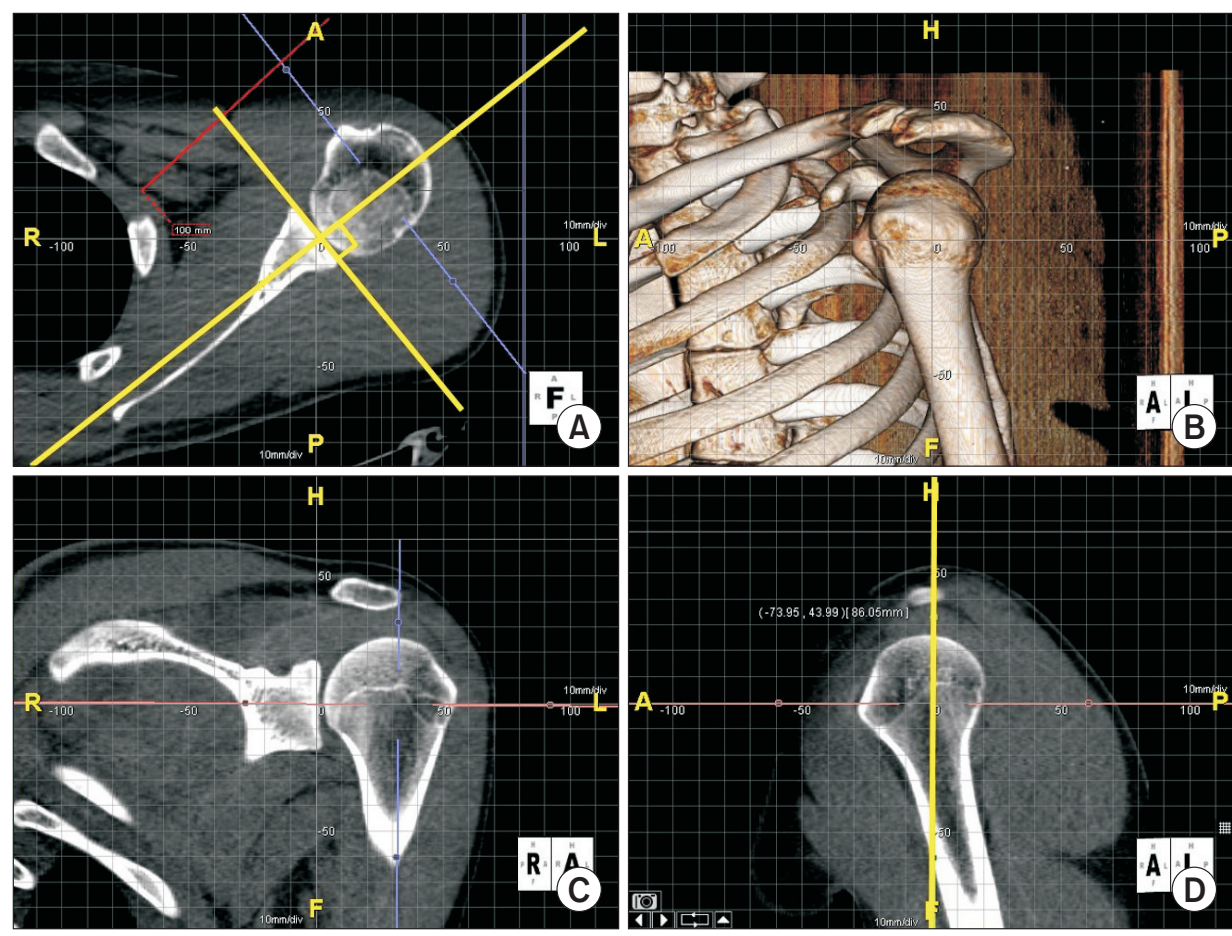

Fig. 4. The most lateral scapular bony structure of the perpendicular plane to glenoid (white line). (A) Axial image: identify the plane that passes through the glenoid center, perpendicular to the tangent line through the glenoid. (B) Three-dimensional reconstructed volume rendering model. (C) Coronal image: sagittal image is moved to the lateral side while viewing the coronal image. (D) Sagittal image: the most lateral side of the perpendicular plane to glenoid passes through the acromion.

Table 1. The Most Lateral Scapular Bony Structure of the Two Planes (the Scapular Plane and the Perpendicular Plane to Glenoid) and the Shortest Distance from the Anterolateral Corner of the Acromion to the Two Planes

\begin{tabular}{lcc}
\hline \multicolumn{1}{c}{ Variable } & $\begin{array}{c}\text { Scapular } \\
\text { plane }\end{array}$ & $\begin{array}{c}\text { Perpendicular plane } \\
\text { to glenoid plane }\end{array}$ \\
\hline $\begin{array}{l}\text { Outermost structure that meets the } \\
\text { plane (\% of patients) }\end{array}$ & 97.0 & 94.0 \\
\hline Acromion & 2.0 & 3.0 \\
\hline Acromioclavicular joint & 1.0 & 3.0 \\
\hline Lateral end of the clavicle & $10.44 \pm 5.11$ & $9.55 \pm 5.13$ \\
\hline $\begin{array}{l}\text { Shortest distance from the anterolat- } \\
\text { eral corner of the acromion to each } \\
\text { plane (mm) }\end{array}$ & & \\
\hline
\end{tabular}

Values are presented as percent only or mean \pm standard deviation.

\section{Results}

The lateral most scapular bony structure of the scapular plane are the acromion ( $97.0 \%$ of patients), the AC joint (2.0\% of patients), and the lateral end of the clavicle (1.0\% of patients). The shortest distance from the anterolateral corner of the acromion to the scapular plane was assessed at $10.44 \pm 5.11 \mathrm{~mm}$. The lateral most structure that the perpendicular plane to glenoid plane first meets is the acromion. The perpendicular plane to glenoid plane passes through the acromion in $94.0 \%$ of patients, AC joint in $3.0 \%$ of patients, and lateral end of the clavicle in $3.0 \%$ of patients. The shortest distance from the anterolateral corner of the acromion to the perpendicular plane to glenoid
Table 2. Distance between the Midpoint of the Fulcrum Axis and the Two Planes (Scapular and Perpendicular Plane to Glenoid)

\begin{tabular}{lcc}
\hline & $\begin{array}{c}\text { Scapular } \\
\text { plane }\end{array}$ & $\begin{array}{c}\text { Perpendicular plane } \\
\text { to glenoid plane }\end{array}$ \\
\hline $\begin{array}{l}\text { Distance between the central point of the } \\
\text { fulcrum axis and each axis }(\mathrm{mm})^{*}\end{array}$ & $3.90 \pm 3.21$ & $3.84 \pm 3.17$ \\
\hline
\end{tabular}

Values are presented as mean \pm standard deviation.

${ }^{*}$ Acromion side: + , coracoid side: - .

plane was $9.55 \pm 5.13 \mathrm{~mm}$ (Table 1). The scapular plane passes at $3.90 \pm 3.21 \mathrm{~mm}$ and the perpendicular plane to glenoid plane passes at $3.84 \pm 3.17 \mathrm{~mm}$ towards the acromion from the midpoint of fulcrum axis, which is defined as a line between the posterolateral corner of the acromion and the tip of the coracoid process (Table 2). In measuring the shortest distance from the most prominent anterolateral corner of the acromion to each plane, inter-observer reliability and intra-observer reliability at the scapular plane are 0.901 and 0.892 , respectively, and 0.938 and 0.928 , respectively, at the perpendicular plane to glenoid plane. For evaluating the distance between the central point of the fulcrum axis and each plane, the inter-observer reliability and intra-observer reliability are 0.849 and 0.869 , respectively, at the scapular plane and 0.841 and 0.843 , respectively, at the perpendicular plane to glenoid plane (Table 3). Our data indicates that all values show excellent agreement. 
Table 3. Inter-observer and Intra-observer Reliability in Evaluating the Shortest Distance from the Anterolateral Corner of the Acromion to Each Plane, and the Distance between the Midpoint of the Fulcrum Axis and Each Planes

\begin{tabular}{lcc}
\hline \multicolumn{1}{c}{ Variable } & $\begin{array}{c}\text { Scapular } \\
\text { plane }\end{array}$ & $\begin{array}{c}\text { Perpendicular plane } \\
\text { to glenoid plane }\end{array}$ \\
\hline $\begin{array}{l}\text { The shortest distance from the anterolateral } \\
\text { corner of the acromion to the axis }\end{array}$ & 0.938 \\
Inter-observer reliability & 0.901 & 0.928 \\
Intra-observer reliability & 0.892 & \\
$\begin{array}{l}\text { Distance between the central point of the } \\
\text { fulcrum axis and each axis }\end{array}$ & \\
Inter-observer reliability & 0.849 & 0.841 \\
\hline Intra-observer reliability & 0.869 & 0.843 \\
\hline
\end{tabular}

\section{Discussion}

The concept of an intraoperative anatomical landmark or a consistent anatomical relationship to scientifically define the orientation of the glenoid surface would allow for a standardized reference of glenoid component preparation, especially for defining the glenoid center and facilitating the insertion of a guide pin through the glenoid center axis. In the current study, the scapular plane passes through the acromion in 97 out of 100 cases $(97.0 \%)$, and the mean of the shortest distances from the anterolateral corner of the acromion to the plane was $10.44 \pm$ $5.11 \mathrm{~mm}$. In addition, the perpendicular plane to glenoid plane passes through the acromion in 94 out of 100 cases (94.0\%), and the mean of the shortest distances from the anterolateral corner of the acromion to the plane was $9.55 \pm 5.13 \mathrm{~mm}$. Barrett et al. ${ }^{28)}$ described the glenoid centerline and the centering point at the anterior glenoid neck as surgical landmarks to determine the glenoid version and degree of correction frequently used in clinical practice. However, their study does not evaluate the accuracy of this method. Our study used a computer program to rearrange the plane and find the scapular plane, and to correct the scapular plane. Moreover, we also used external reference points (the posterolateral corner of acromion and the tip of the coracoid process) which are palpable by the surgeon. Compared to using the scapular neck as a reference point, external reference points are more easily accessible and familiar to surgeons during the surgery. In addition, the reference points used in this study are part of the scapula. Therefore, these points minimize the bias due to the patient position. In the current study, we analyzed the reference point and made quantitative evaluations (such as distance from anatomical landmark like anterolateral corner of the acromion).

Using reference points has some clinical relevance. First, when drawing the center line, the surgeon has two points (the center point and the reference point) that can guide the correct scapular plane. Second, a surgeon can accurately estimate after inserting the drill guide by evaluating the reference point. We used the lateral scapular bony structure of scapular plane or perpendicular plane to glenoid, as well as the 'fulcrum axis' as the external references. ${ }^{7,29,30)}$ The fulcrum axis connects the tip of the coracoid and the posterolateral corner of the acromion. Both surface landmarks are easy to palpate and are familiar to shoulder surgeons. The essential advantage of the fulcrum axis is the use of superficial landmarks which allow intraoperative application of the preoperative planned version of the glenoid using the same landmarks. However, this method does not represent the correct glenoid version but the approximated glenoid version. In contrast, the current study evaluates the point where the scapular plane passes through the fulcrum axis. The scapular plane passes at $3.90 \pm 3.21 \mathrm{~mm}$ towards the acromion from the midpoint of the line between the posterolateral corner of acromion and the tip of the coracoid process. In addition, the perpendicular plane to glenoid plane passes at $3.84 \pm 3.17 \mathrm{~mm}$ towards the acromion from the midpoint of the line between the posterolateral corner of acromion and the tip of the coracoid process. These quantified external reference points may give the surgeon two points which guide the scapular plane when performing centering drilling. Therefore, we can imagine a hypothetical line passing through the posterolateral corner of acromion and the tip of the coracoid process during the surgery, and another line passing through point at about $3.90 \pm 3.21$ $\mathrm{mm}$ toward the acromion at the center of hypothetical line and glenoid center which may be considered as a scapular plane.

There are some limitations in our study. First, this study was carried out only through radiologic evaluations. Therefore, selecting the appropriate computed tomographic slice for evaluation may be difficult and different depending on the observers, thereby affecting the calculated results. However, statistics of the inter-observer and intra-observer reliability studies show excellent agreement. We believe that using a computer program and the plane rearrangement were fundamental in achieving this agreement. Second, the enveloping soft tissue was not considered for this study. Since the distinction between bony surface and soft tissue surface is not definite in the operation field, applying our results to clinical application should be interpreted with care. However, this study suggests the reference and guide. The reference points and results will provide information regarding new techniques, such as the navigation system, as well as provide information for further studies. Third, we only evaluated the posterior wear and did not evaluate the glenoid inclination. Thus, when defining the insertion point, our results only suggest the anterior and posterior direction; we did not suggest the glenoid inclination or superior and inferior direction. However, in cuff tear arthropathy, superior wear is a major problem, whereas posterior wear is the most common type of wear and a challenging problem for the surgeon in osteoarthritis. Hence, further studies are required in this field. Finally, the current study was 
carried out in the Asian population, which means that the results might not represent the global population. However, this study and method may provide some guidelines to further evaluate the external reference in shoulder arthroplasty.

\section{Conclusion}

Our study demonstrates that evaluating the positional relationship between the scapular plane and the surrounding structures by radiographic evaluation is a reliable method. The findings of our study may provide some guidance on glenoid component insertion in shoulder arthroplasty.

\section{References}

1. Strauss EJ, Roche C, Flurin PH, Wright T, Zuckerman JD. The glenoid in shoulder arthroplasty. J Shoulder Elbow Surg. 2009;18(5):819-33.

2. Aaron DL, Flatow EL. Glenoid component design and fixation in total shoulder arthroplasty. Semin Arthroplasty. 2012;23(2):99-102.

3. Gregory TM, Sankey A, Augereau B, et al. Accuracy of glenoid component placement in total shoulder arthroplasty and its effect on clinical and radiological outcome in a retrospective, longitudinal, monocentric open study. PLoS One. 2013;8(10):e75791.

4. Gunther SB, Lynch TL. Total shoulder replacement surgery with custom glenoid implants for severe bone deficiency. J Shoulder Elbow Surg. 2012;21(5):675-84.

5. Clitherow HD, Frampton CM, Astley TM. Effect of glenoid cementation on total shoulder arthroplasty for degenerative arthritis of the shoulder: a review of the New Zealand national joint registry. J Shoulder Elbow Surg. 2014;23(6):775-81.

6. Iannotti JP, Norris TR. Influence of preoperative factors on outcome of shoulder arthroplasty for glenohumeral osteoarthritis. J Bone Joint Surg Am. 2003;85(2):251-8.

7. Braunstein V, Korner M, Brunner U, Mutschler W, Biberthaler P, Wiedemann E. The fulcrum axis: a new method for determining glenoid version. J Shoulder Elbow Surg. 2008;17(5):81924.

8. Papadonikolakis A, Neradilek MB, Matsen FA 3rd. Failure of the glenoid component in anatomic total shoulder arthroplasty: a systematic review of the English-language literature between 2006 and 2012. J Bone Joint Surg Am. 2013;95(24):2205-12.

9. Braunstein V, Kirchhoff C, Ockert B, et al. Use of the fulcrum axis improves the accuracy of true anteroposterior radiographs of the shoulder. J Bone Joint Surg Br. 2009;91(8):1049-53.

10. Sabesan V, Callanan M, Sharma V, lannotti JP. Correction of acquired glenoid bone loss in osteoarthritis with a standard versus an augmented glenoid component. J Shoulder Elbow
Surg. 2014;23(7):964-73.

11. Sears BW, Johnston PS, Ramsey ML, Williams GR. Glenoid bone loss in primary total shoulder arthroplasty: evaluation and management. J Am Acad Orthop Surg. 2012;20(9):60413.

12. Deutsch A, Abboud JA, Kelly J, et al. Clinical results of revision shoulder arthroplasty for glenoid component loosening. J Shoulder Elbow Surg. 2007;16(6):706-16.

13. Flurin $\mathrm{PH}$, Janout $\mathrm{M}$, Roche $\mathrm{CP}$, Wright TW, Zuckerman J. Revision of the loose glenoid component in anatomic total shoulder arthroplasty. Bull Hosp Jt Dis (2013). 2013;71 Suppl 2:6876.

14. Ho JC, Sabesan VJ, lannotti JP. Glenoid component retroversion is associated with osteolysis. J Bone Joint Surg Am. 2013;95(12):e82.

15. Iannotti JP, Greeson C, Downing D, Sabesan V, Bryan JA. Effect of glenoid deformity on glenoid component placement in primary shoulder arthroplasty. J Shoulder Elbow Surg. 2012;21(1):48-55.

16. Farron A, Terrier A, Büchler P. Risks of loosening of a prosthetic glenoid implanted in retroversion. J Shoulder Elbow Surg. 2006;15(4):521-6.

17. Matsen FA 3rd, Clinton J, Lynch J, Bertelsen A, Richardson ML. Glenoid component failure in total shoulder arthroplasty. J Bone Joint Surg Am. 2008;90(4):885-96.

18. Meyer NJ, Pennington WT, Ziegler DW. The glenoid center point: a magnetic resonance imaging study of normal scapular anatomy. Am J Orthop (Belle Mead NJ). 2007;36(4):200-2.

19. Kircher J, Wiedemann M, Magosch P, Lichtenberg S, Habermeyer P. Improved accuracy of glenoid positioning in total shoulder arthroplasty with intraoperative navigation: a prospective-randomized clinical study. J Shoulder Elbow Surg. 2009;18(4):515-20.

20. Nguyen D, Ferreira LM, Brownhill JR, et al. Improved accuracy of computer assisted glenoid implantation in total shoulder arthroplasty: an in-vitro randomized controlled trial. J Shoulder Elbow Surg. 2009;18(6):907-14.

21. Stübig T, Petri M, Zeckey $C$, et al. 3D navigated implantation of the glenoid component in reversed shoulder arthroplasty. Feasibility and results in an anatomic study. Int J Med Robot. 2013;9(4):480-5.

22. Landau JP, Hoenecke HR. Genetic and biomechanical determinants of glenoid version: implications for glenoid implant placement in shoulder arthroplasty. J Shoulder Elbow Surg. 2009;18(4):661-7.

23. Scalise JJ, Codsi MJ, Bryan J, lannotti JP. The three-dimensional glenoid vault model can estimate normal glenoid version in osteoarthritis. J Shoulder Elbow Surg. 2008;17(3):487-91.

24. Shapiro TA, McGarry MH, Gupta R, Lee YS, Lee TQ. Biomechanical effects of glenoid retroversion in total shoulder arthroplasty. J Shoulder Elbow Surg. 2007;16(3):S90-5. 
25. Hsu JE, Ricchetti ET, Huffman GR, lannotti JP, Glaser DL. Addressing glenoid bone deficiency and asymmetric posterior erosion in shoulder arthroplasty. J Shoulder Elbow Surg. 2013;22(9):1298-308.

26. Hoenecke HR Jr, Hermida JC, Dembitsky N, Patil S, D'Lima DD. Optimizing glenoid component position using threedimensional computed tomography reconstruction. J Shoulder Elbow Surg. 2008;17(4):637-41.

27. Hoenecke HR Jr, Hermida JC, Flores-Hernandez C, D'Lima DD. Accuracy of CT-based measurements of glenoid version for total shoulder arthroplasty. J Shoulder Elbow Surg.
2010;19(2):166-71.

28. Barrett WP, Franklin JL, Jackins SE, Wyss CR, Matsen FA 3rd. Total shoulder arthroplasty. J Bone Joint Surg Am. 1987;69(6):865-72.

29. Le SV, Conrad B, Wright TW. Application of the fulcrum axis to estimate the central scapular axis. J Shoulder Elbow Surg. 2014;23(8):1143-9.

30. Budge MD, Lewis GS, Schaefer E, Coquia S, Flemming DJ, Armstrong AD. Comparison of standard two-dimensional and three-dimensional corrected glenoid version measurements. J Shoulder Elbow Surg. 2011;20(4):577-83. 